\title{
Does Variation in Either Age at Start of Therapy or Duration of Therapy Make Chemoprevention with Finasteride Cost- Effective?
}

\author{
Suzanne Biehn Stewart, MD ${ }^{1,{ }^{*}}$, Charles D. Scales Jr., MD ${ }^{1}$, Judd W. Moul, MD ${ }^{1}$, and Shelby \\ D. Reed, PhD $^{2}$ \\ ${ }^{1}$ Division of Urology, Department of Surgery, Duke University Medical Center, Durham, North \\ Carolina \\ 2Duke Clinical Research Institute, Duke University School of Medicine, Durham, North Carolina
}

\section{Abstract}

\begin{abstract}
Background-Incremental cost-effectiveness ratios (ICER) of finasteride for prostate cancer prevention are consistent with estimates beyond \$100,000 per quality-adjusted life-year (QALY). The majority of these analyses are based on chemoprevention starting in men aged 50-55yrs. We sought to evaluate the impact of varying both age at commencement of therapy and length of therapy on the cost-effectiveness of finasteride.
\end{abstract}

\begin{abstract}
Methods-A probabilistic Markov model was designed to estimate lifetime prostate health related costs and quality-adjusted survival for men receiving or not receiving chemoprevention with finasteride. ICERs across scenarios varying age at start of therapy and duration of chemoprevention were compared.
\end{abstract}

\begin{abstract}
Results-The ICER for men starting chemoprevention at age 50 and continuing to age 75 was $\$ 88,800$ per QALY when assuming finasteride causes a constant risk reduction across all tumor grades (base case 1) and $\$ 142,300$ per when assuming a differential treatment effect according to Gleason score (base case 2). When starting age is increased, the ICERs trend downward and nadir at 65 years to $\$ 64,700$ per QALY (base case 1) and $\$ 118,600$ per QALY (base case 2). Altering duration of therapy had minimal impact. Patient-level experiences with finasteride and BPH significantly influenced the cost-effectiveness of chemoprevention.
\end{abstract}

Conclusion-Initiating chemoprevention at ages when prostate cancer incidence is higher improves its cost-effectiveness profile. Only when assuming a constant risk reduction for all tumor grades, did finasteride fall below $\$ 100,000$ per QALY, but this finding was not upheld when accounting for side effects associated with the drug.

\section{Keywords}

chemoprevention; prostate cancer; finasteride; cost-effectiveness analysis; age

\section{Introduction}

In 2003, the Prostate Cancer Prevention Trial (PCPT) revealed that finasteride was an effective agent for prostate cancer chemoprevention, reducing the 7-year prevalence of

\footnotetext{
*Corresponding author: Suzanne Biehn Stewart, Division of Urology, Department of Surgery, Duke University Medical Center, Box 3707, Durham, NC 27710, Tel: 919.668.8101, Fax: 919.684-8794, suzanne.stewart@ duke.edu.

Conflict of Interest: All authors share no financial interest in relation to the work described
} 
prostate cancer by $24.8 \% .^{1}$ This landmark finding generated much discussion over the societal implications of using finasteride as a chemopreventative agent for prostate cancer. One particular focus revolves around the question of whether it would be a cost-effective strategy to provide treatment for all men.

The majority of cost-effectiveness studies have shown that widespread utilization of finasteride for prostate cancer chemoprevention is not an economically sound strategy. Initial analyses found that each additional year of survival gained with use of finasteride would cost $\$ 1.1$ million to $\$ 1.7$ million. ${ }^{2-4}$ Even after incorporating the impact of chemoprevention on quality of life associated with lower rates of BPH, the costeffectiveness of finasteride, although improved, largely remained above commonly cited willingness-to-pay thresholds of $\$ 50,000$ to $\$ 100,000$ per life year saved. ${ }^{5-7}$ The willingness to pay threshold is the amount of money that society has deemed acceptable to pay for one additional year of life in good health. These analyses showed that the incremental costeffective ratio (ICER), a ratio of the incremental costs relative to the incremental benefits afforded by a therapeutic treatment, ranged from $\$ 89,000$ to $\$ 130,000$ per quality-adjusted life-year (QALY). ${ }^{4,8,9}$ The QALY is a function of survival and health-related quality of life added by an intervention. Overall, these studies had assumed that men initiated finasteride at age 50-55 and continued treatment for 10-30 years or until development of prostate cancer or death from other causes.

While the use of finasteride in all men has proven not to be an economically attractive approach, targeting its use to higher-risk groups may be more reasonable. ${ }^{10}$ Reed et al. found that when positive family history and/or higher number of inherited risk alleles for prostate cancer were incorporated, a lower ICER for finasteride could be achieved by targeting higher-risk men. ${ }^{9}$ Earnshaw et al. found that limiting chemoprovention to men with other risk factors such as abnormal DRE, positive family history, or elevated PSA markedly improved the cost-effectiveness of chemoprevention with 5ARIs. ${ }^{10}$ However, if a population-wide chemoprevention strategy is a goal, there may be efficiency gains in delaying chemoprevention until men are at higher risk for prostate cancer or shortening the duration of treatment. Thus, we investigated the cost-effectiveness of using finasteride across scenarios in which we varied both age at start of therapy and duration of chemoprevention.

\section{Methods}

The study design is a cost-effectiveness analysis conducted from a health care system perspective. A probabilistic Markov computer simulation model, developed by Reed et al. ${ }^{9}$, was used to estimate lifetime prostate health related costs and quality-adjusted survival for men receiving or not receiving chemoprevention with finasteride.

\section{Base Assumptions}

The Markov model represents 8 distinct health states. (Figure 1) During each annual cycle, patients could remain cancer free, die from other causes or develop prostate cancer.

Distributions of cancer grades were derived from the PCPT. ${ }^{1}$ Two base case analyses were created to account for differences in treatment effects with finasteride. The first base case analysis (base case--1) reflects a constant risk reduction across all tumor grades of $24.8 \%$ when men are treated with finasteride. The second base case analysis (base case--2) incorporates the influence of tumor grade-specific treatment effects with finasteride, where the risk of low grade tumors are decreased by $38.2 \%$ and the risk of intermediate and high grade tumors are increased by $23 \%$ and $67 \%$, respectively. ${ }^{1}$ Each treatment effect, when evaluated, remained constant throughout the duration of chemoprevention with finasteride although the risk of developing prostate cancer varied with age. 
If patients developed prostate cancer, they were assumed to undergo prostatectomy. Patients who progressed to biochemical recurrence or metastatic disease were assumed to be treated with androgen deprivation therapy. Transition rates to biochemical recurrence, metastatic disease and death were based on validated nomograms ${ }^{11}$ and literature reports concerning the natural history of prostate cancer following radical prostatectomy ${ }^{12}$ and use of androgen deprivation treatment (Table 1). ${ }^{13}$ Patients, not treated with finasteride, who developed benign prostatic hyperplasia (BPH) were treated with alpha-blockers.

\section{Costs, Health State Disutilities, and Cost-Effectiveness Ratios}

Medical costs associated with prostate cancer care from diagnosis to death were based on a recent cost analysis of cancer care in elderly patients (Table 1). ${ }^{14}$ The report by Stewart et al. provided the basis for the utility weights applied to represent quality of life experienced by men with prostate cancer or BPH (Table 2). ${ }^{13} \mathrm{~A} 3 \%$ annual discount rate was applied to all future costs and survival years. Monte Carlo simulations provided the distributions to derive the $95 \%$ confidence intervals (CIs) for the ICERs.

To determine the most efficient age at which to initiate chemotherapy and treatment duration from a cost-effectiveness perspective, we generated ICERs when initiating finasteride at the age of 50 until 70 years, using 5-year increments, and the duration of chemoprevention using 5-year increments up to the age of 75. This cut point was chosen based on the US Preventive Services Task Force recommendation to discontinue screening for prostate cancer in men older than 75 as the harms of screening past this age outweigh the benefits. ${ }^{15,16}$ Sensitivity analyses were performed using both base case analyses to evaluate the impact of individual model parameters and assumptions.

\section{Results}

In our model, the average man at age 50 years has a (remaining) lifetime risk of prostate cancer of $16.1 \%$. Starting finasteride for chemoprevention at this age, and continuing until age 75 , reduced the lifetime risk of prostate cancer to $13.7 \%$ when assuming that finasteride exerts a constant risk reduction across all tumor grades (base case--1) and a 14.5\% lifetime risk when assuming finasteride exerts a differential treatment effect on tumor grades (base case--2). The estimated mean number of QALYs for men starting chemoprevention at age 50 and continuing treatment until age 75 years was estimated as 17.958 (discounted) at a cost of $\$ 13,700$ (discounted) per person in base case -1 and 17.921 (discounted) at a cost of $\$ 14,000$ (discounted) per person in base case-2. In men not receiving finasteride, the discounted mean number of QALYs was 17.855 at a cost of \$4,602 per person. Thus, in base case-1, use of finasteride as chemoprevention starting at age 50 and continuing treatment for 25 years resulted in a gain of 102 QALYs per 1000 men (95\% CI: 58.6-149.6) and an incremental cost-effectiveness ratio of $\$ 88,800$ per QALY (95\% CI: 59,900159,300). In base case-2, use of finasteride starting at age of 50 and continuing for 25 years resulted in a gain of 65.8 QALYs per 1000 men (95\% CI: 31.4-107.1) and an incremental cost-effectiveness ratio of \$142,300 per QALY (95\% CI: 86,400-294,000).

\section{Influence of Starting Age and Duration of Therapy}

In both base case analyses, when age at start of therapy is increased beyond age 50, the ICERs initially trend downward (Figure 2), and nadir at an age of 65 years to $\$ 64,700$ per QALY (base case-1: constant risk reduction) and \$118,600 per QALY (base case-2: differential treatment effect). As age at start of therapy increases, the absolute gain in discounted QALYs saved consistently declines. The cost-effectiveness of chemoprevention improves, however, because incremental costs associated with chemoprevention decrease as the age at initiation is delayed. When duration of therapy is also altered, ICERs less than 
$\$ 100,000$ per QALY were only realized when using a constant risk reduction for finasteride, base case- 1 . Here, the most cost-effective strategies occurred when starting at age 65 and continuing chemoprevention for 5 years (ICER, $\$ 63,800$ per QALY) or 10 years (ICER $\$ 64,700$ per QALY). (Figure 3)

\section{Sensitivity Analysis}

Alteration of utility weights and cost parameters for base case--2, even with the most optimal age and duration strategy, consistently resulted in ICERs greater than $\$ 100,000$ per QALY (data not shown). However, changing these parameters for base case-1, using a starting age of 65 and duration of therapy of 10 years, resulted in some scenarios where the ICER of finasteride fell below $\$ 50,000$. (Figure 4) For example, when increasing the disutility of BPH to 0.1 from the base-case assumption of 0.05 , the ICER for chemoprevention dropped to $\$ 48,900$ per QALY. However, if no disutility for BPH is assumed (utility of 1.0), then the ICER for chemoprevention with finasteride increases to $\$ 95,600$ per QALY. On the other hand, applying a disutility of 0.01 for finasteride, thereby giving some influence to the drug's side effect profile, caused a dramatic shift in the results with a net reduction in the expected QALYs with chemoprevention compared to no chemoprevention ( -34 per 1000 men). Given that expected costs with chemoprevention remained higher, this represented a scenario in which chemoprevention with finasteride was economically dominated. Finally, changing the cost of primary treatment to simulate more expensive radiation treatments (triple the cost) or less expensive active surveillance (halve the cost) had very little impact on the base case estimate of $\$ 64,700$.

\section{Discussion}

Based on our model parameters, ICERs representing chemoprevention with finasteride surpassed $\$ 100,000$ per QALY across all ages for initiation and duration of therapy when assuming that finasteride had a differential treatment effect on tumor grade. Only when assuming a constant risk reduction across all tumor grades were ICERs less than $\$ 100,000$ per QALY. With this assumption, chemoprevention was most cost-effective when therapy was delayed until men were at a higher risk for prostate cancer. Varying the starting age had more impact on the ICER than varying the duration of therapy. For example, delaying the initiation of chemoprevention from age 50 until age 65, using a constant risk reduction for finasteride, resulted in the corresponding ICERs dropping from $\$ 132,000$ per QALY to $\$ 64,000$ per QALY when duration of therapy was 5 years. Prolonging therapy to 10 years when starting at the age of 65 induced little change in the ICER as gains in quality-adjusted survival were commensurate with costs associated with chemoprevention over the longer time period. Overall, these results show that the cost-effectiveness of finasteride can improve when initiated at ages when the incidence of prostate cancer is the highest and duration of use is relatively short. This finding is consistent with analyses that evaluate the cost-effectiveness of chemoprevention in men at higher risk of developing prostate cancer. $9,17-19$

When assuming a constant risk reduction across all tumor grades, the strategy of starting chemoprevention at age 65 and continuing until age 75 was one of the most cost-effective approaches identified. However, even with this strategy, the upper limit of the corresponding 95\% confidence interval $(95 \%$ CI $\$ 45,700-\$ 111,800)$ still extends beyond the willingnessto-pay threshold of $\$ 100,000$ per QALY. Some of the uncertainty stems from variability associated with the utility weights assigned to adjust for differences in quality of life. When holding the utility weights constant, the $95 \%$ confidence interval ranged from $\$ 49,000$ to $\$ 98,400$ per QALY. Thus, for decision makers who want greater certainty that the ICER is less than $\$ 100,000$ per QALY, greater precision about the quality of life experienced by men receiving chemoprevention and men with prostate cancer is needed. 
Our sensitivity analysis showed that the ICER of finasteride was greatly influenced by changes in utility weights associated with BPH and receipt of finasteride. The most dramatic impact occurred when assuming that men receiving finasteride experienced a disutility with treatment. With just a 0.01 disutility assigned to represent potential side effects with finasteride, chemoprevention was expected to provide fewer QALYs than no chemoprevention. This is an important finding as finasteride has been associated with higher rates of erectile dysfunction, abnormal ejaculation and breast enlargement than placebo. ${ }^{1}$ The impact of drug associated disutility has also been found to be highly influential on the cost-effectiveness of tamoxifen as chemoprevention in breast cancer. ${ }^{21}$ Additionally, placement of a greater disutility on BPH symptoms (i.e. -0.1), resulted in the ICER of finasteride falling below $\$ 50,000$ per QALY. Thus, depending on patient level experiences with the drug and its effectiveness in controlling clinically significant BPH, the costeffectiveness of finasteride can markedly improve. Recently, finasteride has been found to reduce the risk of clinically incident BPH in asymptomatic men by $40 \%{ }^{22}$ This effect was represented in our model. Finally, in our model, we assumed that men undergo radical prostatectomy following a prostate cancer diagnosis. We acknowledge that this may not represent treatment preferences for many men. However, we found that varying the cost of primary prostate cancer treatment induced relatively small changes in the cost-effectiveness of finasteride.

One other issue that could influence the cost-effectiveness of chemoprevention is the difference in prostate cancer screening that may occur between patients who receive chemoprevention and those who do not. As there continues to be much discussion surrounding the relationship of finasteride and development of high grade prostate cancer $^{20,23-25}$, it is possible that in practice, patients taking finasteride may be more frequently screened with PSA tests and prostate biopsies. The potential of more frequent PSA lab draws and prostate biopsies in the chemoprevention group would undoubtedly impose a certain degree of disutility for patients taking finasteride, increase costs, and potentially increase the detection of prostate cancer. However, without data on the increase in frequency of screening and the degree of disutility generated, we could not incorporate this potential effect into the model.

\section{Conclusions}

Based on our model, a population-based prostate cancer chemoprevention strategy would not generally be considered cost-effective (i.e. ICERs greater than $\$ 100,000$ per QALY) when assuming that finasteride causes a differential treatment effect on tumor grades. With a willingness to pay threshold of $\$ 100,000$ per QALY, only when applying a constant risk reduction across all tumor grades could chemoprevention with finasteride be considered cost-effective. Delaying chemoprevention until men are at the ages when the incidence of prostate cancer is higher could provide a more efficient strategy for a population-wide chemoprevention program. Nevertheless, consideration of patient-level experiences with finasteride and BPH will ultimately guide personalized risk-benefit decisions for initiating chemoprevention. As shown in our analyses, the cost-effectiveness of chemoprevention will become more attractive if it is more often prescribed for men at higher risk of developing prostate cancer, in men with $\mathrm{BPH}$, and in men who do not experience significant side effects associated with finasteride.

\section{Acknowledgments}

Supported in part by research funds from the Committee for Urologic Research, Education, and Development (CURED) of Duke University and grant RC2CA148463 from the National Cancer Institute. The content of this manuscript is solely the responsibility of the authors and does not necessarily represent the official views of the National Cancer Institute or the National Institutes of Health. 


\section{References}

1. Thompson IM, Goodman PJ, Tangen CM, et al. The influence of finasteride on the development of prostate cancer. N Engl J Med. 2003; 349:215-24. [PubMed: 12824459]

2. Svatek RS, Lee JJ, Roehrborn CG, Lippman SM, Lotan Y. The cost of prostate cancer chemoprevention: a decision analysis model. Cancer Epidemiol Biomarkers Prev. 2006; 15:1485-9. [PubMed: 16896037]

3. Svatek RSLJ, Roehrborn CG, et al. Erratum: the cost of prostate cancer chemoprevention: a decision analysis model. Cancer Epidemiol Biomarkers Prev. 2007; 1:1042.

4. Zeliadt SB, Etzioni RD, Penson DF, Thompson IM, Ramsey SD. Lifetime implications and costeffectiveness of using finasteride to prevent prostate cancer. Am J Med. 2005; 118:850-7. [PubMed: 16084177]

5. Laupacis A, Feeny D, Detsky AS, Tugwell PX. How attractive does a new technology have to be to warrant adoption and utilization? Tentative guidelines for using clinical and economic evaluations. CMAJ. 1992; 146:473-81. [PubMed: 1306034]

6. Hornberger JC, Redelmeier DA, Petersen J. Variability among methods to assess patients' wellbeing and consequent effect on a cost-effectiveness analysis. J Clin Epidemiol. 1992; 45:505-12. [PubMed: 1588356]

7. Hirth RA, Chernew ME, Miller E, Fendrick AM, Weissert WG. Willingness to pay for a qualityadjusted life year: in search of a standard. Med Decis Making. 2000; 20:332-42. [PubMed: 10929856]

8. Svatek RS, Lee JJ, Roehrborn CG, Lippman SM, Lotan Y. Cost-effectiveness of prostate cancer chemoprevention: a quality of life-years analysis. Cancer. 2008; 112:1058-65. [PubMed: 18186497]

9. Reed SD, S JC, Stewart SB, Sun J, Moul JW, Schulman KA, Xu J. Effects of family history and genetic polymorphism on the cost-effectiveness of chemoprevention with finasteride for prostate cancer. J Urol. 2010

10. Earnshaw SR, McDade CL, Black LK, Bell CF, Kattan MW. Cost effectiveness of 5-alpha reductase inhibitors for the prevention of prostate cancer in multiple patient populations. Pharmacoeconomics. 2010; 28:489-505. [PubMed: 20196623]

11. Kattan MW, Eastham JA, Stapleton AM, Wheeler TM, Scardino PT. A preoperative nomogram for disease recurrence following radical prostatectomy for prostate cancer. J Natl Cancer Inst. 1998; 90:766-71. [PubMed: 9605647]

12. Pound CR, Partin AW, Eisenberger MA, Chan DW, Pearson JD, Walsh PC. Natural history of progression after PSA elevation following radical prostatectomy. JAMA. 1999; 281:1591-7. [PubMed: 10235151]

13. Stewart ST, Lenert L, Bhatnagar V, Kaplan RM. Utilities for prostate cancer health states in men aged 60 and older. Med Care. 2005; 43:347-55. [PubMed: 15778638]

14. Yabroff KR, Lamont EB, Mariotto A, et al. Cost of care for elderly cancer patients in the United States. J Natl Cancer Inst. 2008; 100:630-41. [PubMed: 18445825]

15. Stephenson AJ, Scardino PT, Eastham JA, et al. Preoperative nomogram predicting the 10-year probability of prostate cancer recurrence after radical prostatectomy. J Natl Cancer Inst. 2006; 98:715-7. [PubMed: 16705126]

16. Lin K, Lipsitz R, Miller T, Janakiraman S. Benefits and harms of prostate-specific antigen screening for prostate cancer: an evidence update for the U.S. Preventive Services Task Force. Ann Intern Med. 2008; 149:192-9. [PubMed: 18678846]

17. Zeliadt SB, Ramsey SD. Cost-effectiveness of prostate cancer chemoprevention among high-risk men. Expert Rev Pharmacoecon Outcomes Res. 2010; 10:505-8. [PubMed: 20950065]

18. Kattan MW, Earnshaw SR, McDade CL, Black LK, Andriole GL. Cost effectiveness of chemoprevention for prostate cancer with dutasteride in a high-risk population based on results from the REDUCE clinical trial. Appl Health Econ Health Policy. 2011; 9:305-15. [PubMed: 21875161] 
19. Svatek RS, Lotan Y. Cost utility of prostate cancer chemoprevention with dutasteride in men with an elevated prostate specific antigen. Cancer Prev Res (Phila). 2011; 4:277-83. [PubMed: 21163937]

20. Shepherd BE, Redman MW, Ankerst DP. Does Finasteride Affect the Severity of Prostate Cancer? A Causal Sensitivity Analysis J Am Stat Assoc. 2008; 103:1392-404.

21. Noah-Vanhoucke J, Green LE, Dinh TA, Alperin P, Smith RA. Cost-effectiveness of chemoprevention of breast cancer using tamoxifen in a postmenopausal US population. Cancer. 2011; 117:3322-31. [PubMed: 21404271]

22. Parsons JK, Schenk JM, Arnold KB, et al. Finasteride Reduces the Risk of Incident Clinical Benign Prostatic Hyperplasia. Eur Urol. 2012

23. Redman MW, Tangen CM, Goodman PJ, Lucia MS, Coltman CA Jr, Thompson IM. Finasteride does not increase the risk of high-grade prostate cancer: a bias-adjusted modeling approach. Cancer Prev Res (Phila). 2008; 1:174-81. [PubMed: 19138953]

24. Lucia MS, Epstein JI, Goodman PJ, et al. Finasteride and high-grade prostate cancer in the Prostate Cancer Prevention Trial. J Natl Cancer Inst. 2007; 99:1375-83. [PubMed: 17848673]

25. Cohen YC, Liu KS, Heyden NL, et al. Detection bias due to the effect of finasteride on prostate volume: a modeling approach for analysis of the Prostate Cancer Prevention Trial. J Natl Cancer Inst. 2007; 99:1366-74. [PubMed: 17848668]

26. Bosch JL, Hop WC, Kirkels WJ, Schroder FH. Natural history of benign prostatic hyperplasia: appropriate case definition and estimation of its prevalence in the community. Urology. 1995; 46:34-40. [PubMed: 7544515]

27. [Accessed August 28, 2009] at http://www.drugstore.com/pharmacy/ 


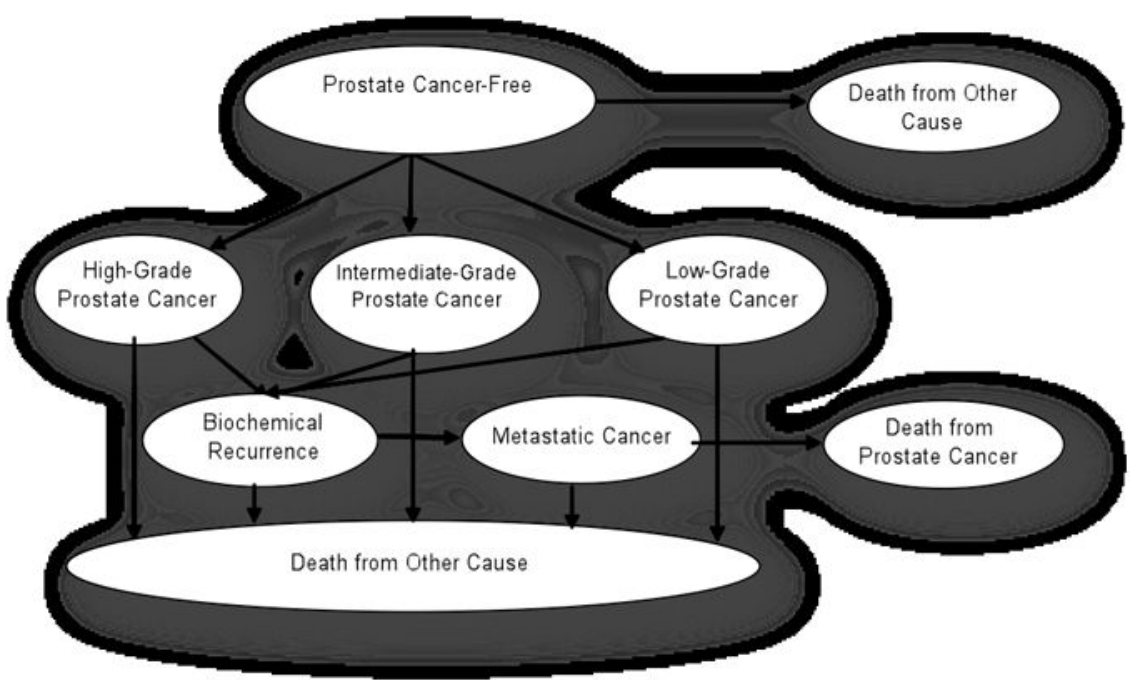

Figure 1. Markov model and 8 distinct health states

Caption: All patients are initially assumed to be free of prostate cancer. On an annual cycle, patients can remain in the same health state or transition to one of the seven other states.

Transition to death not attributable to prostate cancer can occur from any health state. 


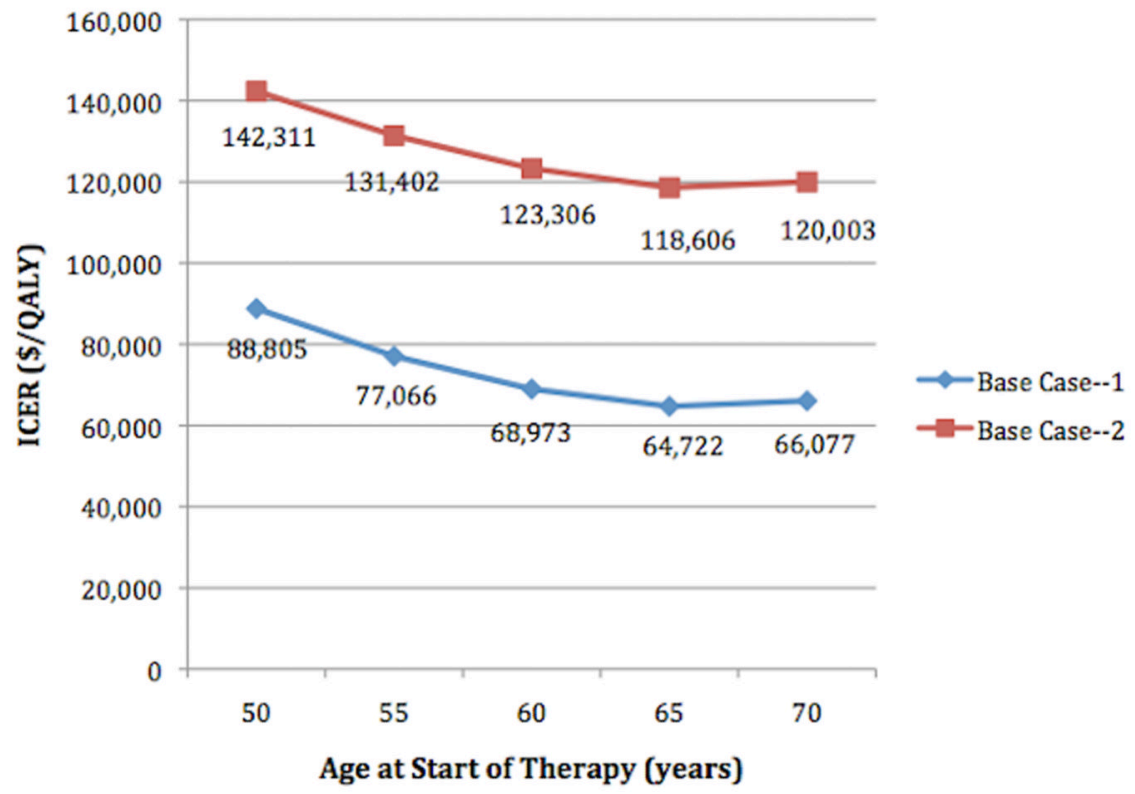

Figure 2.

Variation in Age at Start of Chemoprevention and Continuation until Age 75 using both a Constant Risk Reduction (Base case-1) and a Differential Treatment Effect (Base case-2) by Finasteride across Prostate Cancer Grades.

Caption: Base Case--1 reflects a relative risk reduction of 0.752 across all Gleason Scores and Base Case--2 reflects a differential treatment effect according to Gleason Scores (GS): GS 2-6 = 0.6186; GS $7=1.2306$; GS 8-10 = 1.6709. Abbreviations: ICER, incremental costeffective ratio; QALY, quality-adjusted life-year. 


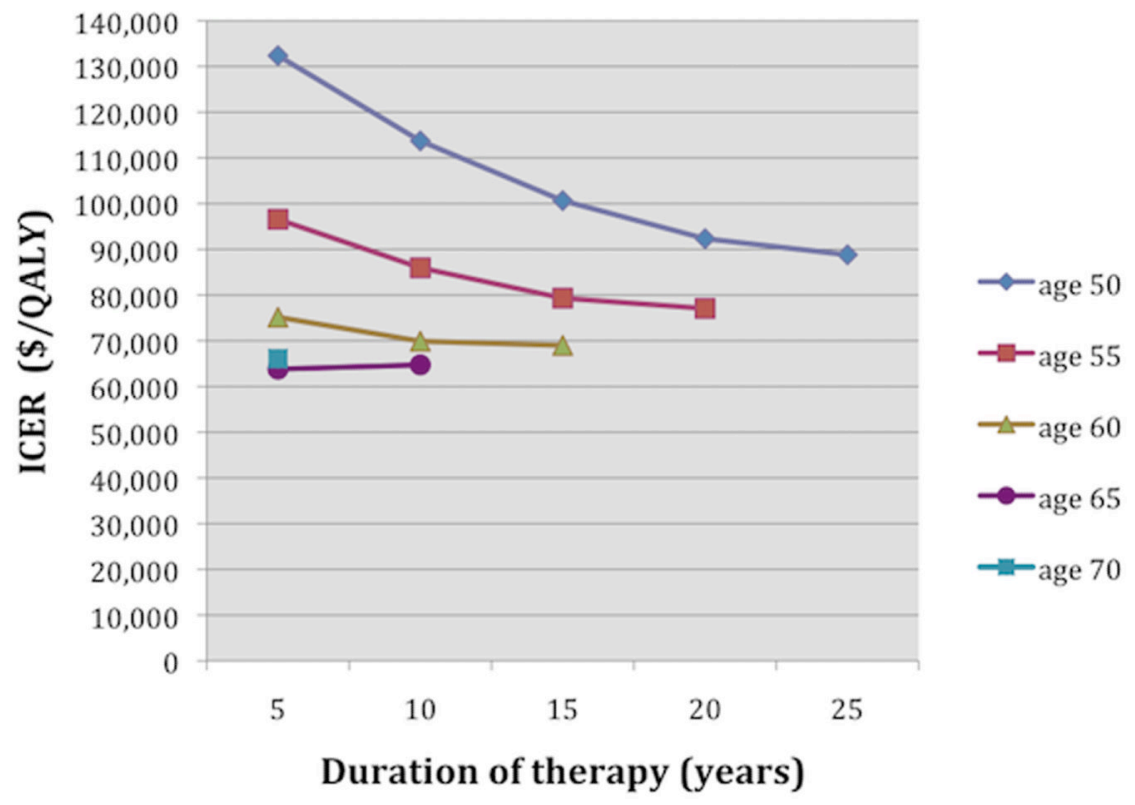

Figure 3.

Influence of Duration of Chemoprevention at Different Starting Ages of Therapy.

Abbreviations: ICER, incremental cost-effective ratio; QALY, quality-adjusted life-year. 


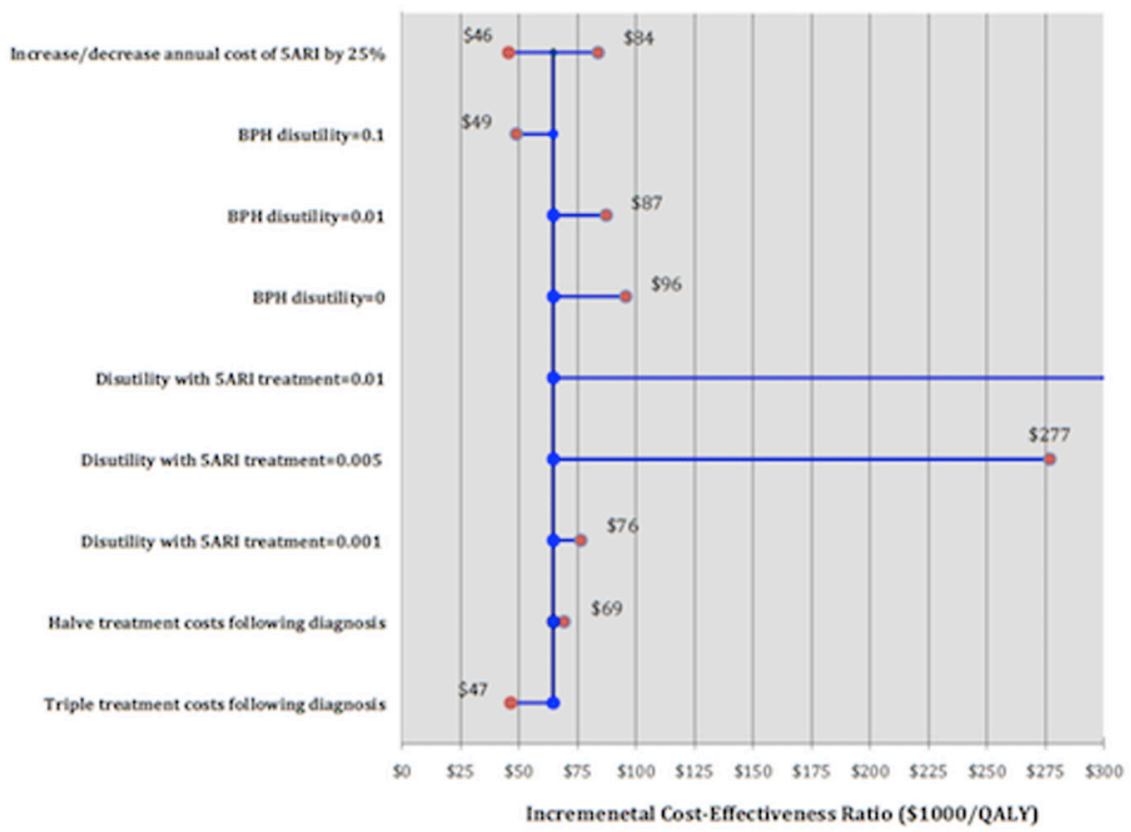

Figure 4. One-way Sensitivity Analysis

Abbreviations: BPH, benign prostatic hyperplasia; QALY, quality-adjusted life-year. Caption: A 1-way sensitivity analysis varying model parameters and assumptions for the base case estimate of starting finasteride at age 65 years and continuing therapy for 10 years. Longer bars indicate a greater sensitivity of the cost-effective ratio with that parameter. The single line extending beyond the $\mathrm{x}$-axis when using a disutility of 0.01 with 5-ARI treatment represents an economically dominated scenario (ie, higher costs and fewer quality-adjusted life-years). 
Table 1

Model Parameters and Base Assumptions

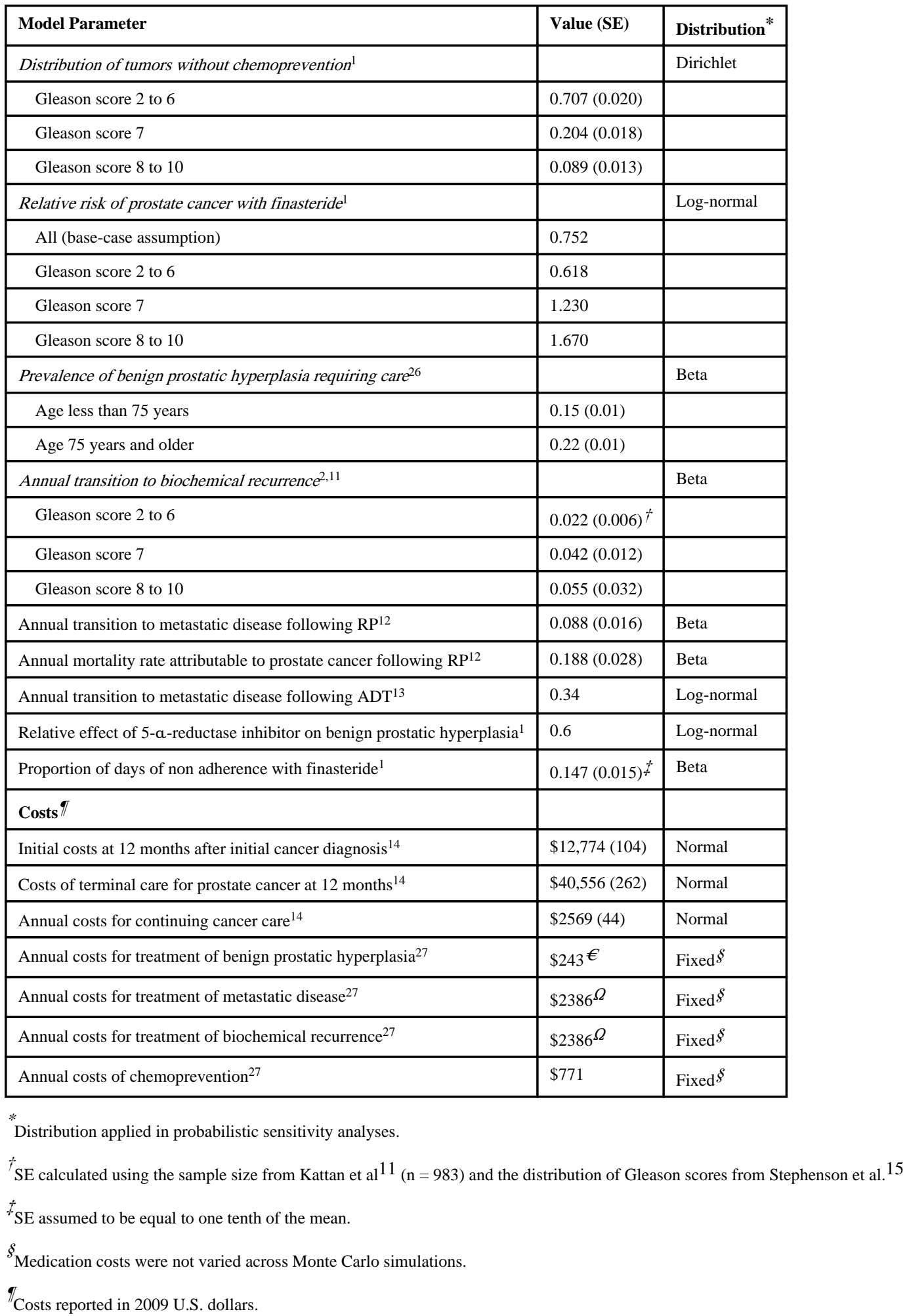

Prostate Cancer Prostatic Dis. Author manuscript; available in PMC 2013 December 01. 
$€_{\text {Based on doxazosin } 4 \mathrm{mg} \text { daily, if not being treated with finasteride }}$

$\Omega$ Based on leuprolide $7.5 \mathrm{mg}$ monthly 
Health State Disutilities ${ }^{13}$

Table 2

\begin{tabular}{|l|l|l|}
\hline Disutility & Value (SE) & Distribution $^{*}$ \\
\hline After prostatectomy & & Beta \\
\hline Gleason score 2 to 6 & $0.16(0.02)$ & \\
\hline Gleason score 7 & $0.19(0.03)$ & \\
\hline Gleason score 8 to 10 & $0.29(0.03)$ & \\
\hline Biochemical recurrence & $0.33(0.04)$ & Beta \\
\hline Metastatic disease & $0.75(0.01)$ & Beta \\
\hline Treated benign prostatic hyperplasia & $0.05(0.02)$ & Beta \\
\hline Treatment with 5-a-reductase inhibitor & 0 & Beta \\
\hline
\end{tabular}

$\Psi$ Disutility represents a reduction from a state of perfect health (utility $=1$ ).

Distribution applied in probabilistic sensitivity analyses. 